\title{
Medical versus surgical treatment of ectopic pregnancy
}

\author{
Gladys C. AL JASHI ${ }^{1}$, Andra BORDEIANU ${ }^{2}$, Massawi TARIG ${ }^{3}$, Dimitrie C. NANU ${ }^{4}$, Isam AL JASHI ${ }^{5,2}$, \\ 1"St. Pantelimon“ Emergency Hospital, Bucharest, Romania \\ ${ }^{2}$ Faculty of Medicine, "Titu Maiorescu" University, Bucharest, Romania \\ ${ }^{3}$ Ilfov County Clinical Hospital, Bucharest, Romania \\ 4"Carol Davila" University of Medicine and Pharmacy, Bucharest, Romania \\ 5"Regina Maria“ Private Care Clinic, Bucharest, Romania
}

\begin{abstract}
Ectopic pregnancy is defined as the pregnancy that implants outside the endometrial cavity and is considered a medical emergency.

The objective of the study was to evaluate and compare the results obtained in patients diagnosed with ectopic pregnancy treated with medication versus those treated surgically and to analyze the most effective treatment method by comparing the results obtained.

Material and methods. We conducted a retrospective study on a group of 70 patients diagnosed with ectopic pregnancy at the Ilfov County Clinical Hospital in Bucharest, Romania, over a period of four years. We analyzed each patient, clinically hemodynamic stable or unstable, the percentage of healed patients and those who were pregnant after medical and surgical treatment.

Results. From the total of 70 patients, $83 \%$ of them were hemodynamically stable, $17 \%$ were hemodynamically unstable. Most $87 \%$ of the patients were treated with methotrexate and the remaining $12 \%$ required surgery. Patients treated with methotrexate were completely healed, and $60 \%$ of them got pregnant in the same uterine tube where the ectopic pregnancy occurred.

Conclusions. Clinical trials have demonstrated a 95\% to 100\% rate of ectopic pregnancy resolution through medical therapy. The goal is to avoid anesthesia and surgery, because surgical therapy is an invasive method by which the woman can become infertile, so it is reserved for hemodynamically stable patients. In conclusion, through medical treatment, patients maintains their fertility and may got pregnant in the same tube where the ectopic pregnancy occurred.
\end{abstract}

Keywords: ectopic pregnancy, hemodynamically stable, fertility, methotrexate

\section{INTRODUCTION}

Ectopic pregnancy occurs when the implantation of the fertilized egg occurs elsewhere than in the uterus, most commonly in the fallopian tubes. $95 \%$ of ectopic pregnancies are implanted in various segments of the fallopian tube: $70 \%$ ampul- lary, $12 \%$ isthmic, $11 \%$ fimbria, $2-3 \%$ interstitial, $2 \%$ cornice. The remaining $5 \%$ of non-tubular tasks are implanted in: ovary, peritoneal cavity, cervix, scarring following cesarean surgery.

Incidence in Northern Europe increased in 1976 and 1993 from 11.2 to 18.8 per 1,000 cases (1), and in 1989 their number increased from 
17,800 in 1970 to 88,400 in US hospitals (2). In the Great Britain, about 11,000 cases of ectopic pregnancy occur yearly (1).

Ectopic pregnancy is considered a medical emergency, the most common complication that occurs is rupture of the fallopian tube and tubal abortion. Due to modern methods of diagnosis and treatment the patient can be treated before the symptoms and signs appear.

Determination of $\beta$-HCG serum levels together with transvaginal ultrasound helped improve diagnosis, although there is a controversy regards $\beta$-HCG as a diagnostic method $(3,4)$. During ectopic pregnancy, $\beta-H C G$ is lower than in the intrauterine ones. In a normal pregnancy, the concentration of this hormone doubles every 2 to 3 days from the 4 th day to the 8 th day of gestation (5). The rise in $\beta-H C G$ levels suggests an extrauterine pregnancy. Serum progesterone is used for differential diagnosis with intrauterine pregnancy, so a value less than $5 \mathrm{ng}$ identifies the ectopic pregnancy.

Ectopic pregnancy involves two treatment methods: medical and surgical. Drug treatment is done with methotrexate in a single dose, $50 \mathrm{mg} / \mathrm{m}^{2} /$ $\mathrm{kg}$ body surface area and more doses of $1 \mathrm{mg} / \mathrm{kg} /$ body weight in days $1,3,5$, and 7 . This treatment is recommended for stable hemodynamic patients and the surgical treatment for hemodynamically unstable patients. Surgery is performed laparoscopically either by conserving the uterine tube, salpingostomy, or by remove it by salpingectomy or classic laparotomy.

If the patient is earlier diagnosed, than exist a lower risk of mortality, so this can exclude the invasive investigations, thus allowing the noninvasive therapeutic approach.

The objective of the study was to evaluate and compare the results obtained in patients diagnosed with ectopic pregnancy treated medical versus surgical, to analyze the evolution of patients who had been cured after methotrexate treatment and those remaining pregnant in the same fallopian tube were the ectopic pregnancy was, the follow-up in time of recurrent ectopic pregnancies in the same uterine tube and the evaluation of the most effective treatment method by comparing the results obtained.

\section{MATERIALS AND METHODS}

We conducted a retrospective study on a group of 70 patients diagnosed with ectopic pregnancy at the Ilfov County Clinical Hospital in Bucharest, Romania, over a period of four years from January 2014 to December 2018, of which 65 were treated medical and 9 surgical.

For all patients we noted: age, if the patient was smoking, if they were stable or unstable hemodynamic, number the days of hospitalization, if the ectopic pregnancy was complicated or uncomplicated, the paraclinical diagnostic methods used, visualisation of the adnexal mass on the transvaginal ultrasound, the size of the adnexal mass, the location of the ectopic pregnancy, the contraceptive methods used, the personal history of the patient, the number of patients medical and those treated surgical, the patients who were pregnant depending on what type of treatment had been used, those who were pregnant in the same fallopian tube, recurrence of extrauterine pregnancy on the same tube, complications and side effects after drug treatment. The information has been extracted from the anamnesis and then was processed as tables and graphs in Microsoft Office Excel.

\section{RESULTS}

Ectopic pregnancy is the major cause of maternal morbidity and mortality. The highest incidence in this study was between the ages of 30 and 45 , representing $80 \%$, and $14 \%$ had aged between 25 and 28 years, out of a total of 70 patients. The youngest age was 45 , while the youngest was 25 years old.
FIGURE 1. Clinical manifestations at patients with ectopic pregnancy

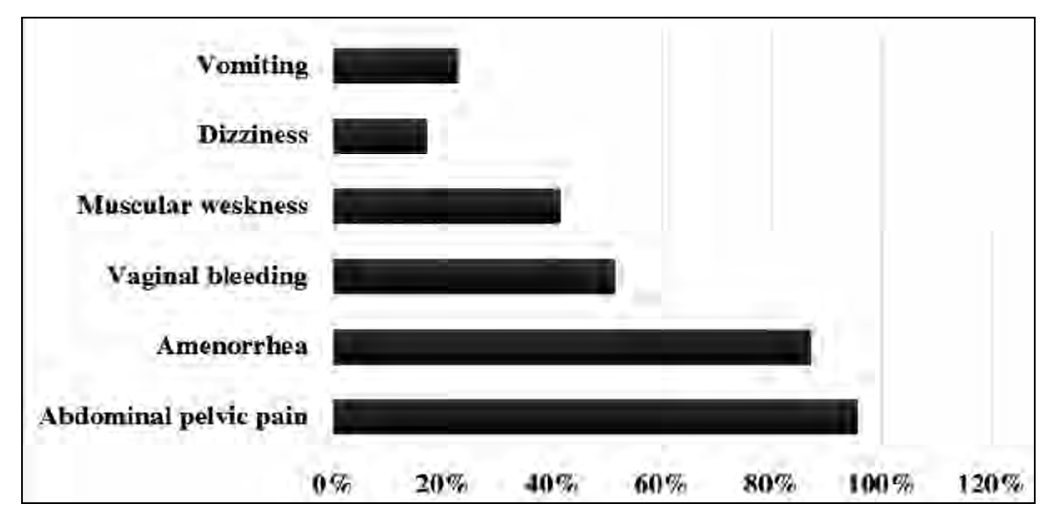


TABLE 1. Paraclinical diagnostic method

\begin{tabular}{|l|c|c|c|c|c|c|}
\hline $\begin{array}{l}\text { Paraclinical } \\
\text { diagnostic method }\end{array}$ & $\begin{array}{c}\text { Positive } \\
\text { pregnancy test }\end{array}$ & $\begin{array}{c}\text { Transvaginal } \\
\text { ultrasound }\end{array}$ & $\begin{array}{c}\text { B-hCG and serum } \\
\text { progesterone levels }\end{array}$ & Culodocentesis & Laparoscopic & Total \\
\hline Number of cases & 69 & 61 & 52 & 6 & 3 & 70 \\
\hline Percentage & $66 \%$ & $87 \%$ & $74 \%$ & $9 \%$ & $4 \%$ & $100 \%$ \\
\hline
\end{tabular}

From all 70 patients registered, $69 \%$ of the them were smokers and the other of $31 \%$ were non-smokers.

The results from this study are in line with those in the literature so patients presented with the following signs and symptoms: of a total of 70 patients, the main manifestation was pelvic abdominal pain in $96 \%$ of patients, followed by amenorrhea to $87 \%$ of these, $51 \%$ had vaginal bleeding, 41\% muscle weakness, $23 \%$ vomiting and $17 \%$ dizziness (Fig. 1 ).

In the retrospective study, we counted of patients the hospitalization days, as well as patients progression after medical or surgical treatment. Out of the 70 patients, $74 \%$ were discharged in less than 7 days, $11 \%$ of the patients were discharged from 7 to 10 days, and $14 \%$ more than 10 days.

Of the total of 70 patients, $83 \%$ were hemodynamically stable and $17 \%$ hemodynamically unstable.

In the case of patients with broken or unbroken ectopic pregnancy symptoms are not very obvious. Following the analysis of the 70 patients, the majority of $87 \%$ experienced uncomplicated ectopic pregnancy, while the remaining $6 \%$ had complicated ectopic pregnancy.

From the data extracted, $27 \%$ of the patients presented a positive pregnancy test, $36 \%$ of them had transvaginal ultrasound. Determination of $\beta$-hCG levels and serum progesterone levels were performed in $31 \%$ of women, culodocentesis in $4 \%$ of patients with broken ectopic pregnancy. Laparoscopy was required in $3 \%$ of the 70 patients be- cause transvaginal ultrasound was inconclusive (Table 1).

From 70 patients, transvaginal ultrasound was performed on 61 of them, adnexal mass were seen in the right tube on 37 patients, while in 19 of them the location was on the left and in 5 of them no adnexal mass was observed.

From 61 patients which had been done transvaginal ultrasound, was observed in 58 of the patients an adnexal mass between 2 and $4 \mathrm{~cm}$, while in the remaining 3 patients the adnexal mass could not be detected.

Most ectopic pregnancies were located at the fallopian tube and that was on $94 \%$ of the patients, while in $4 \%$ of them the ectopic pregnancy was cervical and only in one patient the ectopic pregnancy was corneal.

Many patients, $41 \%$ used oral contraceptives, $21 \%$ used intrauterine devices, $15 \%$ mentioned condom use, and $11 \%$ did not use any contraceptive method.

In the patients with ectopic pregnancy, the highest percentage of $24 \%$ resulted in patients with a history of vaginal infection, then $16 \%$ of them had pelvic inflammatory disease, a major risk factor in the occurrence of ectopic pregnancy. Other history of pathology and associated diseases: hypertension in $13 \%$ of patients, $11 \%$ diabetes mellitus, 9\% obesity, history of ectopic pregnancy and endometriosis $7 \%$, genital surgery $6 \%, 4 \%$ infertility treatment and malformations congenital uterus (uterus retrovers) in 3\% (Fig. 3).

For most of the patients diagnosed with ectopic pregnancy methotrexate was administered, in

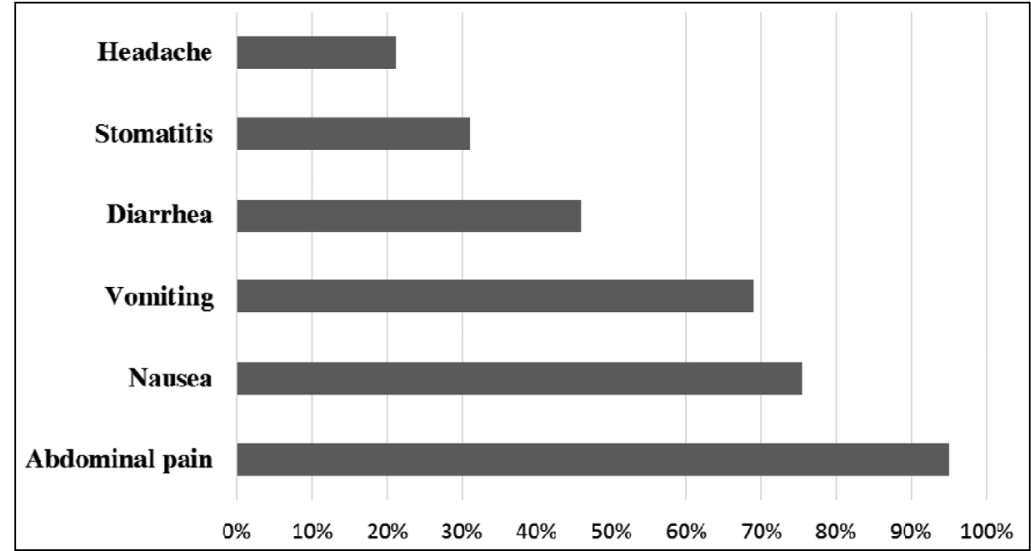




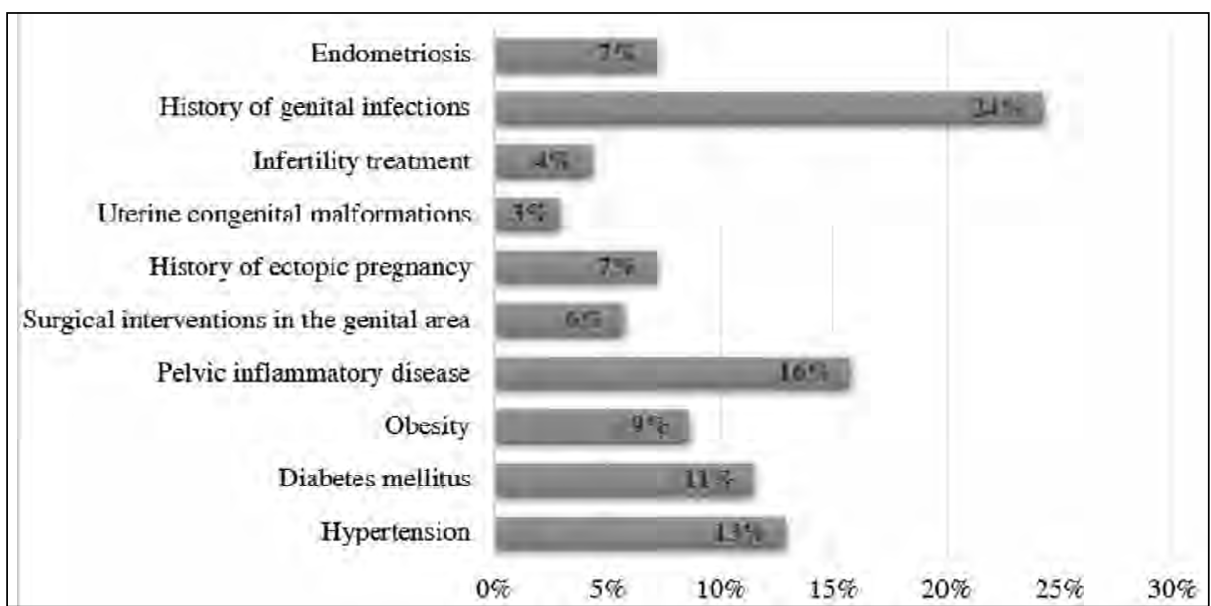

FIGURE 3. Pathological personal antecedents and related diseases

TABLE 2. Type of treatment

\begin{tabular}{|l|c|c|c|c|c|}
\hline $\begin{array}{l}\text { Type of } \\
\text { treatment }\end{array}$ & MTX & $\begin{array}{c}\text { Laparoscopic } \\
\text { Salpingostomy }\end{array}$ & $\begin{array}{c}\text { Laparoscopic } \\
\text { Salpingectomy }\end{array}$ & Laparotomy & Total \\
\hline Number of cases & 61 & 5 & 1 & 3 & 70 \\
\hline Percentage & $87 \%$ & $7 \%$ & $1 \%$ & $4 \%$ & $100 \%$ \\
\hline
\end{tabular}

$87 \%$, while the remaining $12 \%$ required surgery, laparoscopic salpingostomy was performed at $7 \%$ and laparoscopic salpingectomy was performed on one, while laparotomy was required in the remaining 4\% (Table 2).

As a result of the medical treatment, we noticed that all 61 patients treated with methotrexate healed completely, that means a percentage of $87 \%$ patients and after the surgical treatment $10 \%$ had been completely healed, while in $3 \%$ of them their condition improved.

After treatment with methotrexate $60 \%$ remain pregnant from a total of 61 healed patients and from 9 patients that needed surgery, 33\% remain pregnant (Fig. 4).

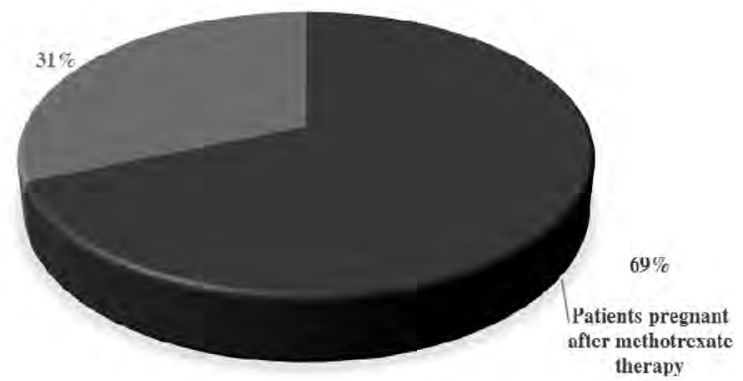

FIGURE 4. Patients pregnant after methotrexate therapy

From 70 cases, 61 were treated with methotrexate and after treatment, a total of 42 patients remain pregnant, of whom 25 were pregnant in the same uterine tube where the ectopic pregnan- cy occurred, representing a percentage of $60 \%$, the remaining 17 patients were pregnant in the healthy uterine tube (Table 3 ).

TABLE 3. Patients who got pregnant after methotrexate in the same fallopian tube where the ectopic pregnancy was

\begin{tabular}{|l|c|c|c|}
\hline $\begin{array}{l}\text { Patients who got pregnant } \\
\text { after Methotrexate in the } \\
\text { same fallopian tube where } \\
\text { the ectopic pregnancy was. }\end{array}$ & $\begin{array}{c}\text { Pregnant } \\
\text { in the } \\
\text { same } \\
\text { tube }\end{array}$ & $\begin{array}{c}\text { Pregnant } \\
\text { in the } \\
\text { opposite } \\
\text { tube }\end{array}$ & Total \\
\hline Number of cases & 25 & 17 & 42 \\
\hline Percentage & $60 \%$ & $40 \%$ & $100 \%$ \\
\hline
\end{tabular}

Ectopic pregnancy has a relapse risk and from 70 patients with a positive ectopic pregnancy diagnosis, at $4 \%$ of them recurred after surgery. While there was no recurrent ectopic pregnancy in those treated with Methotrexate.

Various side effects may occur following the administration of Methotrexate. Of the 70 patients, 61 patients had the following side effects: severe abdominal pain $95 \%$, nausea $75 \%$, vomiting $69 \%$, diarrhea $46 \%$, stomatitis $31 \%$, headache $21 \%$ (Fig. 2).

\section{DISCUSSION}

Diagnosis of ectopic pregnancy is confirmed most often before symptoms and specific signs appear and due to modern paraclinical investigations: serological tests and transvaginal ultrasound. 
Treatment of ectopic pregnancies involves two types of approaches: a surgical and medical approach, depending on the condition of the patient at the time of diagnosis.

Methotrexate is folic acid antagonist, it can be administrated single or in multiple doses, while the surgical treatment involve laparoscopic therapy or laparotomy.

Methotrexate in ectopic pregnancy prevents proliferation of cytotrophoblast cells by reducing cell viability and $\beta$-hCG synthesis but also progesterone, thus ectopic pregnancy is removed. It can also be applied also in corneal and cervical ectopic pregnancies, which has been reported successfully in the international literature $(6,7)$.

The first case that it was treated with methotrexate occurred in 1987 in a study by Feichtinger and Kemeter (8).

In 1988, Leeton and Davison reported two cases that were diagnosed with uncomplicated and non-complicated extrauterine pregnancy, which were successfully healed by administering methotrexate (9).

Epidemiological statistics have shown that smoking is a major risk factor in the occurrence of ectopic pregnancy due to nicotine that affects the function of the fallopian tubes. From the data extracted, it was observed that the majority of $69 \%$ of the patients are smokers.

The specific triad for ectopic pregnancy, consisting of pelvic abdominal pain, amenorrhea and vaginal bleeding, was reported in 96\%, $87 \%$ and $51 \%$ from 70 cases analysed. A clinical trial indicates that pelvic pain was reported in $90 \%$ of women, amenorrhea to $81 \%$ of patients, and vaginal bleeding to $78 \%$ (10). From that, we can say that it is in agreement with what we found in our study.

As a paraclinical diagnostic method, transvaginal ultrasound, serum $\beta$-hCG and progesterone levels and the pregnancy test were used in $66 \%$ of cases. Despite the international statistics were the pregnancy test were $100 \%$ positive. Transvaginal ultrasound is the best diagnostic method for extrauterine pregnancies, in this study it was used in $87 \%$ of cases.

Other diagnostic methods used were: serum $\beta-h C G$ and progesterone levels in $74 \%$ of cases.

In the case of hemodynamically stable patients with adnexal mass less than $4 \mathrm{~cm}$, as identified by transvaginal ultrasound, $\beta$-hCG below 10,000 U/ $\mathrm{ml}$ and without fluid in the peritoneal cavity, drug therapy may be used (11). Thus, from 70 cases, $87 \%$ of them used methotrexate therapy and the remaining $13 \%$ were surgically treated. Both types of treatment have been successful in this study. Methotrexate treated patients had a $100 \%$ success rate and all the patients have been fully healed.

For patients who needed surgical therapy, laparoscopic salpingostomy was performed in $7 \%$ of them, salpingostomy is a procedure that preserve the fallopian tube, while laparoscopic salpingectomy remove uterine tube. In our study was required for single patient. Laparotomy was necessary in $4 \%$ of patients, which were unstable hemodynamically. The success rate of drug and surgical treatment are similar with other statistics $(12,13)$.

In terms of fertility statistics have shown that both types of treatment are good ways of preserving fertility. In our study $60 \%$ of women treated with methotrexate remain pregnant, while the percentage for those treated surgically was lower, from 9 patients, 3 of them remained pregnant.

There is an important connection between $\beta$-hCG serum levels and treatment with methotrexate due to the risk of broken ectopic pregnancy if the $\beta$-hCG level increases after administration of methotrexate.

Side effects of methotrexate are different, but not significant, in our study we registered values closer to other studies from the literature, such as: $95 \%$ of the cases reported abdominal pain, $75 \%$ nausea and $69 \%$ vomiting.

There are many statistics showing the benefits of medical therapy compared to surgery, where complications can be numerous. Among the most important are: hemorrhage, thrombi, infections, visceral damage or in tissues. Therefore, it is important that patients present themselves to the doctor in advance to prevent complications.

\section{CONCLUSIONS}

Ectopic pregnancy is a common complication of pregnancy, which occurs frequently in young women in their fertile periods with an increased risk of morbidity and mortality.

At the moment, there is a possibility to establish the correct diagnosis before the symptoms appear, so the treatment with fewer side effects can be applied and complications can be prevented.

A correct diagnosis, in terms of ectopic pregnancy, should be performed by repeated transvaginal ultrasounds.

Medical therapy with methotrexate is a safe and effective method for treating ectopic pregnancies.

Over time, the mortality rate in patients with ectopic pregnancy has declined considerably due 
to modern and accessible paraclinical investigations, diagnosis and treatment.

Clinical trials have demonstrated a $95 \%$ to $100 \%$ rate of resolution in ectopic pregnancy through drug treatment.

The treatment of ectopic pregnancies will be determined according to the signs and symptoms of the patient, transvaginal ultrasound and serum $\beta$-hCG levels. Methotrexate is indicated for all hemodynamically stable patients without broken ectopic pregnancy and low $\beta$-hCG below 5,000 mIU/ $\mathrm{mL}$. Patients with negative Rh will need administration of immunoglobulin anti-D.
As regards the evolution of patients with history of ectopic pregnancy, this remains unfavorable with high risk of recurrence.

The goal is to avoid anesthesia and surgery because the surgical procedure is an invasive method during which the woman can remain infertile, so this is reserved for stable hemodynamic patients.

In conclusion, through medical treatment, patients maintain their fertility and may become pregnant on the same fallopian tube where the ectopic pregnancy occurred.

\section{Conflict of interest: none declared} Financial support: none declared

\section{REFERENCES}

1. Storeide O, Veholmen M, Eide M, Bergsjo $\mathrm{P}$, Sandvei R. The incidence of ectopic pregnancy in Hordal and County, Norway 1976-1993. ActaObstet Gynecol Scand 1997. p. 76:345-349.

2. Centers for Disease Control and Prevention. Ectopic pregnancy - United States, 1990-1992. MMWR Morb Mortal WklyRep 1995. p. 44:46-48.

3. Mehta TS, Levine D, Beckwith B. Treatment of ectopic pregnancy: Is a human chorionic gonadotropin level of $2,000 \mathrm{mlU} / \mathrm{ml} \mathrm{a}$ reasonable threshold? Radiology 1997. p. 205:569-573.

4. Mol BWJ, Hajenius PJ, Engelsbel S et al. Serum human chorionic gonadotropin measurement in the diagnosis of ectopic pregnancy when transvaginal sonography is inconclusive. Fertil Steril 1998. p. 70:972-981.

5. Pittaway DE, Reish RL, Wentz AC. Doubling times of human chorionicgonadotropin increase in early viable intrauterine pregnancies. AmJ Obstet Gynecol 1985. p. 152:299-302.

6. Brandes MC, Youngs DD, Goldstein DP, Parmley TH. Treatment of cornual pregnancy with methotrexate: case report. Am J Obstet Gynecol; 1986. p. 155:655.

7. Kaplan BR, Brandt $T$, Javaheti $G$, Scommegna A. Nonsurgical treatment of a viable cervical pregnancy with intraamniotic methotrexate. Fertil Steril; 1990. p. 53:941.

8. Feichtinger W, Kemeter P. Conservative treatment of ectopic pregnancy by transvaginal aspiration under sonographic control and methotrexate injection. Lancet 1987. p.1:381.

9. Leeton J, Davison G. Nonsurgical management ofunruptured tubal pregnancy with intra-amniotic methotrexate: prelimi- nary report of two cases. Fertil Steril; 1988 p. 50:167.

10. National Institutes of Health. Ectopic pregnancy comparison of different treatments. https://www.ncbi.nlm.nih.gov/ pmc/articles/PMC3279172/ (Accessed on May 28, 2019).

11. National Institutes of Health. Clinical Analysis of Ectopic Pregnancies in a Tertiary Care Centrein Southern India: A Six-Year Retrospective Study. https://www. ncbi.nlm.nih.gov/pmc/articles/ PMC5121740/\#b1 (Accessed on May 28, 2019).

12. National Institutes of Health. Medical treatment of ectopic pregnancy with methotrexate. https://www.ncbi.nlm.nih.gov/ pubmed/9341620 (Accessed on May 28, 2019).

13. National Institutes of Health. Randomized trial of conservative laparoscopic treatment and methotrexate administration in ectopic pregnancy and subsequent fertility https:// www.ncbi.nlm.nih.gov/ pubmed/9853887?dopt=Abstract (Accessed on May 28, 2019). 